\title{
Heinz-Gerhard Haupt \\ Sind Mittelklassen Mittelklassen? Handwerker und Kleinhändler in Frankreich zwischen 1850 und 1914.
}

»Ich bin heute der Sprecher dieser Mittelklasse, dieses produktiven Kleinbürgertums unserer Städte und Dörfer, das sich organisiert, Gewerkschaften bildet und sich zusammenschließt, weil es sich verteidigen will und weil es leben will. Aber, Herr Präsident, auch wenn es sich über seine Interessen und Rechte eindeutiger klar wird, vergißt es nicht, was es der nationalen und sozialen Solidarität, dem Vaterland und der Demokratie schuldig ist. (Lebhafter Beifall) «. (Etudes fiscales, 1910: 388)

In diesem Toast, den Maurice Colrat auf dem 3. Kongreß der »classes moyennes«Frankreichs im Jahre 1910 ausbrachte, drückte er das Selbstverständnis der organisierten Kleinhändler und Handwerker aus. Diese verstanden sich als Klasse zwischen dem Bürgertum und dem Proletariat, zwischen »dem Kapital und den Arbeitern« (La Revendication, 25.1.1890) und benutzten als Bezeichnung, um ihren Platz in der Gesellschaft zu kennzeichnen, den Begriff, mit dem das Bürgertum seine Ansprüche gegen den Adel durchgesetzt hatte: die Mittelklasse (la classe moyenne). (Fischer, 1974) Demgegenüber konnten sich Termini wie Kleinbürgertum oder die von Léon Gambetta favorisierte Bezeichnung "couches nouvelles« nicht durchsetzen. (Barral, 1968, 232; Dubois, 1962, 14-16) Am Ende des 19. Jahrhunderts und vor allem zu Beginn des 20. Jahrhunderts nahmen sich die Orgaanisationen des Kleinhandels und Handwerks als Zusammenschlüsse der Mittelklassen wahr. So bildeten sie 1907 eine "Vereinigung zur Verteidigung der Mittelklassen«, während die von den Ideen Le Plays inspirierte Zeitschrift La Réforme sociale im Jahre 1910 eine Konferenz über das Schicksal der mclasses moyennes« abhielt und französische Delegierte an dem »Internationalen Kongreß der Mittelklassen« teilnahmen. (Saint-Léon, 1911, 372)

Mit der Selbstbezeichnung waren bestimmte Konsequenzen verbunden. Nicht nur orientierten sich die Kleinbürgerverbände am bürgerlichen Vorbild und entlehnten ihren Namen dem bürgerlichen Emanzipationskampf, sondern sie behaupteten auch, daß zwischen Handwerk und Kleinhandel keine so tiefgreifenden Unterschiede bestünden, die es untersagten, sie als Mitglieder einer Klasse zu verstehen. Darüber hinaus wurde diese im nationalen Zusammenhang und an der Seite der Demokratie verankert. Aus der Mittelklasse zwischen Kapital und Lohnarbeit wollte die Klasse ihre mäßigende Wirkung entfalten, zwar genug bürgerliche Werte aufnehmen, sich aber sehr wohl vom kapitalistischen Bürgertum distanzieren und in der Verbindung von Kapital und persönlicher Mitarbeit gar eine klassenüberwindende Kraft ausbilden.

Die Wirklichkeit einer Klasse geht in ihrem Selbstverständnis nicht auf. Aber dieses muß doch ernst genommen werden, wenn sich Klassenanalysen nicht nur auf der objektiv-strukturellen Ebene bewegen sollen und wenn die Selbsteinschätzung überdies eine Begrifflichkeit benutzt, die auch als analytische Kategorie gelten kann. Deshalb soll von dem in Verlautbarungen von Organisationen verbreiteten Mittelklassenbewußtsein ausgegangen und gefragt werden, ob die Handwerker und Kleinhändler durch ökonomische Bedingungen, soziale Kontakte und Bezüge und politisches Engagement zu einer Klasse geeint oder ob sie durch 
deutliche Unterschiede getrennt waren. Wenn ihre Klassenlage, Klassenbeziehungen und - position bestimmt sind, dann kann angegeben werden, ob die Zusammenfassung der Handwerker und Kleinhändler zur Klasse abgeschlossen ist, welche Veränderungen an der Klassenbegrifflichkeit notwendig sind und ob der Begriff Klasse überhaupt tauglich ist, um die Entwicklung des Kleinbürgertums zu fassen. Damit rückt die Untersuchung ab von entwicklungslogisch angelegten Studien des Kleinbürgertums, die in der Bundesrepublik zumindest publizistische Resonanz fanden (Leppert-Fögen, 1973). In diesen wurde von der Einheit der Klasse ausgegangen und diese lediglich in einem vor allem theoretisch konstruierten gesamtgesellschaftlichen Rahmen verortet. So leitete Leppert-Fögen die Neigung des deutschen Kleinbürgertums zum Faschismus in Anlehnung an Ernst Bloch daraus ab, daß sie in der Weimarer Republik verelende und gleichzeitig der Produktionssphäre fern stünde. Da nur dort jedoch der ungleichzeitige, dem Kapital letztlich nicht gefährliche Widerspruch zu einem gleichzeitigen und revolutionären werden könne, seien die kleinen Bürger aufgrund ihrer »Zwischentätigkeiten« der »gesellschaftlichen Kontinuität« enthoben und in »ein(en) alogische(n) Raum« gewiesen, der den »feuchtwarmen Humus für Ideologie«, für "Wünsche und Romantizismen, Urtriebe und Mytiszismen« abgebe (Bloch, 1973, 108-110). Dieser Ansatz wird weder den historischen Unterschieden zwischen Kleinhändlern und Handwerksmeistern gerecht noch dem Problem, ob und in welchem Maße Kleinbürger für den Faschismus anfällig waren. Neuere Untersuchungen raten gerade zur Vorsicht vor einer vorschnellen Gleichsetzung von Kleinbürgertum und Nationalsozialismus. Da in der kurz vorgestellten Untersuchung weder die Binnenstruktur und Erscheinungsweisen des Kleinbürgertums noch seine Bezüge zu anderen sozialen Klassen bekannt sind, ist A. LeppertFögens Arbeit eher ein intelligenter Entwurf als eine überzeugende Analyse.

Im folgenden wird auf die Unterscheidung zwischen der Klassenlage, der gesellschaftlichen Position und dem politisch-ideologischen Verhalten zurückgegriffen, die Jürgen Kocka für die Untersuchung der deutschen Arbeiterklasse in den ersten beiden Dritteln des 19. Jahrhunderts entwickelt hat (Kocka, 1983). Dieser analytische Rahmen hat den Vorteil, daß er mehrere, die Physiognomie und Bedeutung einer Klasse ausmachende Faktoren berïcksichtigt, mit der sozialen Lage der gesellschaftlichen Erscheinungsform und den gesellschaftlichen Mechanismen stärkere Bedeutung zuweist und den Entwicklungsstand einer Klasse zwar daran mißt, inwiefern zwischen den drei Ebenen eine Gleichläufigkeit und Abhängigkeit besteht, diese aber nicht als notwendig voraussetzt. Überdies können auf allen drei Bereichen die jeweiligen Stellungseigenschaften einer Klasse (Pierre Bourdieu), d.h. die Charakteristika, die aus der Beziehung zu anderen Klassen herrühren, bestimmt werden.

Der Blick auf das französische Kleinbürgertum gewinnt für die Debatte um die deutsche Entwicklung Relevanz, gilt Frankreich doch gemeinhin als das Land, in dem die Krise der Mittelschichten nicht zu einer »Panik im Mittelstand (Theodor Geiger) geführt hat, die dann die "Machtergreifung« des Nationalsozialismus erlaubte. Durch die Erfahrung einer erfolgreichen bürgerlichen Revolution, den schnelleren Abbau von vorindustriellen Traditionen und Leitbildern habe sich das Kleinbürgertum in Frankreich eher an die radikale Partei als an faschistische Gruppierungen angelehnt und sei selbst in seinen irrationalsten Äußerungen noch »populistisch « geblieben (Winkler, 1976). Gegen diese in Vergleichen pauschale Betrachtungsweise wird im folgenden innerhalb des Kleinbürgertums zu differenzieren und zu betonen sein, welche Vielfalt an Lebensweisen und Artikulationsformen sich hinter den allgemeinen Begriffen verbarg. Der komparative Ansatz wird damit von der nationalen auf die regionale oder lokale Ebene verwiesen. 


\section{Expansion und ökonomische Differenzierung der Mittelklassen}

Die Klassenlage der »classes moyennes« ist seit 1848 einmal durch eine zahlenmäßige Expansion, zum anderen durch eine interne Differenzierung gekennzeichnet. Im Gegensatz zu allen pessimistisch-katastrophischen Untergangsprognosen nahmen Handwerksbetriebe und Läden in der zweiten Hälfte des 19. Jahrhunderts in Frankreich zu. Die Zahl derjenigen, die der Gewerbesteuer unterlagen und die im Tableau A vermerkt waren, stieg zwischen 1845 und 1910 um ein Viertel, das Steueraufkommen um die Hälfte und die durchschnittliche Gewerbesteuer von 21,95 auf 35,50 Francs (Landry, 1914, 20-25). Da eine Reihe von Steuerreformen die kleinen Werkstätten und Läden von der Gewerbesteuer ausnahmen, war das zahlenmäßige Wachstum von Handwerk und Kleinhandel noch größer als es die Steuer angibt. Dieser Befund mag auf den ersten Blick befremden, gilt Frankreich doch gemeinhin als Land der langsamen Industrialisierung und wird für diese das große Gewicht der Kleinproduktion angeführt. In einem Vergleich mit anderen europäischen Gesellschaften ist in den letzten zehn Jahren jedoch herausgearbeitet worden, daß sich die französischen Wachstumsraten von den britischen in der zweiten Hälfte des 19. Jahrhunderts nicht wesentlich unterschieden und daß das wirtschaftliche Wachstum vor allem von kleinen und mittleren Betrieben bewirkt wurde. Die Durchsetzung des Kapitalismus fand in Frankreich nicht durch die Verbreitung der großbetrieblichen Strukturen, sondern durch die Unterordnung kleiner und mittlerer Betriebe unter Kapitalinteressen statt. Nicht die »economic backwardness« (Gerschenkron), sondern der spezifisch französische Weg zum Kapitalismus kann die Expansion der Kleinproduktion erklären (O'Brien/Keyder, 1978).

In diesem Wachstumsprozeß traten aber Unterschiede auf. Handwerkliche Arbeit und Handelsgeschäfte wuchsen schneller als kleine industrielle Betriebe, die ländlichen Regionen machten eine besondere Entwicklung durch und Handels- und Handwerksaktivitäten trennten sich.

Während kommerzielle und handwerkliche Betriebe zunahmen, gingen kleinindustrielle Werkstätten zurück. Dieser Schluß kann aus der Tatsache gezogen werden, daß am Ende des 19. Jahrhunderts die Zahl der im Tableau $C$ der Gewerbesteuer verzeichneten Firmen sank, während sich die Kapitalintensität der Betriebe erhöhte und daß wahrscheinlich die kleinen und kapitalschwachen Unternehmen den mittleren und größeren unterlagen (Faure, 1980). Beispiele für diesen Verdrängungswettbewerb boten die katalaunischen Hütten, die in den 1860 er Jahren der großen Verhüttungsindustrie wichen wie auch die Einführung von Maschinen in die Baumwollspinnereien, die kleine Betriebe ruinierten (Armengaud, 1961; Fohlen, 1956). Die Zukunft der Kleinbetriebe lag eher im handwerklichen und kommerziellen als im industriellen Bereich. Vor allem für die Kleinhändler war das 19. Jahrhundert eine goldene Zeit. Durch die Verbesserung der Transportbedingungen und den Rückgang der Selbstversorgung nahmen die der Gewerbesteuer unterworfenen Läden schlagartig zu. Die Zahl der Cafébesitzer versechsfachte sich, die der Kolonialwarenhändler vervierfachte sich, die der Schlachter verdreifachte sich, schließlich verdoppelte sich die Zahl der Bäcker und Gastwirte (Faure).

Wenn auch um 1900 gewerbliche Arbeit auf dem Land noch fortbestand und immer noch ein Drittel der Geschäfte, die gewerbesteuerpflichtig waren, in Orten mit weniger als 2000 Einwohnern bestanden, begann die Bedeutung gewerblicher Aktivitäten außerhalb der Städte seit 1880 abzunehmen. Der Rückgang der landwirtschaftlichen Einkommen und die Schwierigkeit, den Wettbewerb mit größeren und städtischen Gewerben aufrechtzuerhalten, die 
Mechanisierungswelle und die Einigung des nationalen Marktes verschlechterten die Situation der gewerblichen Produktion auf dem Land und führten dazu, daß zahlreiche Handwerker in die Städte abwanderten. So nahm die Zahl der Hutmacher im Departement Loire deutlich ab, gaben Textilproduzenten in Beaujolais die Arbeit auf und starb die Leinenindustrie in dem Heimgewerbe Nordfrankreichs. Vornehmlich im Textil- und Metallgewerbe waren die Veränderungen tief. Sie zeigten sich jedoch weniger im Nahrungsmittel- und für die Landwirtschaft arbeitenden Gewerbe. Trotz aller Strukturwandlungen blieben handwerkliche Betriebe auf dem Lande heimisch (Farcy).

In der zweiten Hälfte des 19. Jahrhunderts trennten sich zunehmend gewerbliche und kommerzielle Aktivitäten. Noch 1848 waren Kleinhändler häufig auch Produzenten. Sie produzierten etwa $17 \%$ des Schinkens, den sie selbst verkauften, wie auch $23 \%$ der Schokolade und rösteten $77 \%$ des von ihnen verkauften Kaffees. Auf der anderen Seite verkauften auch Handwerker ihre Produkte selbst und verbanden Werkstatt und Laden. Deshalb hatten die Statistiker der Jahrhundertmitte noch Schwierigkeiten, zwischen Handels- und Industriefunktionen zu unterscheiden. So wurden in Paris etwa Bäcker noch als Industrielle, Schlachter als Kaufleute wahrgenommen (Faure, 1984; Gaillard, 1977, 430-435). Nach 1848 lösten sich die beiden Funktionen voneinander, ohne daß allerdings die Trennung vollständig war.

Schließlich machten Handwerk und Kleinhandel zu unterschiedlichen Zeiten ihre Erfahrung mit der Kapitalisierung. Das Vordringen des Handelskapitalismus zwang schon in der Julimonarchie Schneider, Schuhmacher und Bauhandwerker ihre Selbständigkeit aufzugeben und abhängige Arbeit zu verrichten. Diese Entwicklung setzte sich fort und beschleunigte sich im 2. Kaiserreich vor allem in den Städten (Scott, 1984; Johnson, 1979, 65-84). Kleinhändler hingegen machten erst nach 1880, in kleineren Städten sogar erst nach 1900 ihre Erfahrung mit großkapitalistischen Distributionsformen. Stammten die ersten Warenhäuser auch aus der Zeit des 2. Kaiserreichs, so breiteten sich erst am Jahrhundertende die Versandgeschäfte und Kettenläden über die großen Städte und aufs Land aus (Gaillard, 1977, 525-58; Gemahling, 1912, 170-192). Diese Phasenverschiebung erklärt, daß am Ende des 19. Jahrhunderts der Kleinhandel der aktivere Teil der »classes moyennes« ist und daß der Begriff Mittelklassen erst dann auf Handwerk und Kleinhandel angewandt wurde, als sich deren Existenzbedingungen annäherten.

Wichtiger als diese Unterschiede waren die internen Differenzierungen in Handwerk und Kleinhandel. Einer Mehrheit von kleinen, kapitalschwachen, auf die Mitarbeit der Familie angewiesenen Werkstätten und Läden stand eine Minderheit von kapitalstarken, unabhängigen Handwerkern und Ladenbesitzern gegenüber. Nach der Berufszählung des Jahres 1901 waren Dreiviertel aller gewerblichen Betriebe Frankreichs entweder Heimarbeiter, Alleinmeister oder Familienbetriebe. Von dem Viertel aller Firmen, die überhaupt familienfremde Arbeitskräfte beschäftigten, hatten $80 \%$ zwischen einem und fünf Angestellten. Auch im Kleinhandel griffen nur $30 \%$ aller Läden auf familienfremde Arbeiter zurück (Faure). Während also die überwiegende Mehrheit der Handwerker und Kleinhändler mitarbeiten mußte, konnte eine kleine Zahl sich auf Aufsicht bzw. gelegentliches Zuarbeiten beschränken. In den meisten Ländern und Werkstätten waren Produktion bzw. Distribution und Reproduk. tion verquickt, nahm die Familie an Verkauf und Herstellung aktiv teil. Nur eine Minderheit konnte sich den Luxus einer Trennung von Familienleben und Erwerbsarbeit leisten. Die Unterschiede treten deutlich bei einem Vergleich der Kapitalien auf, die für eine Ladeneröffnung notwendig sind wie auch bei einem Blick auf das Erbe, das Kleinbürger hinterließen. Im Paris der Mitte des 19. Jahrhunderts wurden durchschnittlich 19260 Francs für den Er- 
werb einer Bäckerei, 2040 für den eines Manufakturwaren- und 700 für den eines Kolonialwarenladens verlangt. Während der Kolonialwarenladen mithin mit Ersparnissen eines Arbeiters erworben werden konnte, setzten die anderen Läden schon die Kombination des Einkommens verschiedener Familienmitglieder bzw. Erbschaften voraus. Bezogen auf die Gesamtzahl der Handwerker und Kleinhändler blieb der Anteil derjenigen, die überhaupt bei ihrem Tode etwas hinterließen, gering. Unbedeutend war er bei Krämern und Fruchthändlern, etwas höher bei Textil-, Haushaltswaren- und Weinhändlern, am höchsten bei Bäckern und Schlachtern. Während diese, zu denen je nach Stadt oder Ortschaft Bauhandwerker oder Textilgeschäfte traten, eine "bourgeoisie populaire (A. Daumard) bildeten, die Krisen überleben, Langzeitplanung anstellen und eine solide Berufsausbildung erwerben konnten, lebte das Gros der Handwerker und Kleinhändler unter "proletaroiden« Bedingungen (T. Geiger).

Diese wurden auch dadurch betont, daß ihre Unabhängigkeit sich oft als eine Fiktion entpuppte. Im Kleinhandel spielten Großhändler oft die Funktion eines Kreditgebers, der die Detaillisten dadurch an sich band, während bereits Kettenläden wie die Docks rémois oder Casino die Krämer in ihrem Ein- und Verkaufsverhalten bestimmten und beschränkten. Auch im Handwerk waren jene selten, die frei unter verschiedenen Rohstofflieferanten und Abnehmern wählen konnten. Für Paris hat Alain Cottereau ihren Anteil an der Gesamtheit der handwerklichen Betriebe für das Jahr 1901 auf nur 1,9\% geschätzt (Cottereau, 69 ff.). Kapitaleinsatz, generell materielle Sicherheit, Selbständigkeit und Familienmitarbeit waren in Handwerk und Kleinhandel höchst unterschiedlich. Zwischen einer Mehrheit, die mit unsicheren Zukunftsperspektiven, geringem Kapital und hoher Selbstausbeutung überlebte und einer Minderheit, die über Kapital, Wohlstand und Freizeit verfügte, schoben sich sicherlich Zwischenpositionen. Aber sie ändern nichts an der ökonomischen Differenzierung der »classes moyennes«, durch die sich auch die Klassenlage und der Erfahrungshorizont unterschiedlich gestaltete.

\section{Soziale Differenzierung und Mobilität der Mittelklassen}

Die Existenzbedingungen von Handwerkern und Kleinhändlern sollen daraufhin untersucht werden, ob enge soziale Beziehungen die beiden Berufsgruppen zu einer Klasse zusammenschlossen und ob das Kleinbürgertum wirklich eine Mittelposition in der französischen Gesellschaft einnahm. Wie dicht die sozialen Kontakte zwischen Gruppen waren, läßt sich an ihrem Heiratsverhalten (so schon Tocqueville, II, 1, 148) und den Kontakten sowohl am Arbeitsplatz als auch am Wohnort erfassen.

Wenn auch neuere Untersuchungen ergeben haben, daß die Wahl einer Frau aus der gleichen Schicht für das Kleinbürgertum in Paris nicht die überragende Bedeutung hatte(Le Yaouang, 1979, 100-102; Corbin, 1981, 113), die Alexis de Toqueville ihr generell zuschrieb, so war die Vermählung doch eine wichtige Entscheidung, die über die spätere Karriere des Mannes mitentschied. Für das Paris der Julimonarchie hat etwa Adeline Daumard ermittelt, daß die Frauen von Ladeninhabern oft aus einer höheren sozialen Schicht stammten als ihre Männer und daß vor allem unter Schlachtern und Bäckern die Sitte verbreitet war, Frauen aus der » moyenne bourgeoisie« zu ehelichen. Ein Drittel aller Ehefrauen von Boutiquiers stammten allerdings aus der Welt des Ladens (Daumard, 1963, 257-259). In Rouen heirateten zu Beginn des 19. Jahrhunderts Krämer und Textilhändler zwar auch in die »gute« Bourgeoisie ein, aber sie verheirateten sich stärker als in Paris mit Arbeiter- und Angestelltentöchtern. Im 2. Kaiser- 
reich suchten sie weiterhin ihre Frauen unter Lohnarbeitern, während ihre Verbindungen mit dem Bürgertum vollends abrissen (Chaline, 1970, 260-264). Daß in der Mitte des Jahrhunderts sich die bürgerlichen Kreise zunehmend gegenüber den kleinen Bürgern abschotteten, erweist Le Yaouang auch für Paris, wo er » weitgehende Verbindungsbereiche« zwischen Kleinbürgern und Arbeitern feststellte (Le Yaouang, 1981, 16; Guillaume, 1972, 122-130). Grenzte sich zu Beginn des 20. Jahrhunderts das Kleinbürgertum Orléans auch nach »unten« ab (Prost, 1981, 679-683), deuten die vorliegenden Forschungen darauf hin, daß Handwerk und Handel vor allem für Verbindungen mit Angestellten- und Arbeiterkreisen offen blieben. Die Emphase, mit der Maurice Colrat 1910 ausrief: "... man sollte wissen, daß auch wir die Hoffnung des Proletariats sind.«(Les Etudes fiscales et sociales, 1911, 411), hatte mithin eine gewisse Berechtigung.

Während die Analyse der Heiratsverhalten die soziale Öffnung des Kleinbürgertums betont, könnten die Beziehungen am Arbeitsplatz einen Hinweis auf die soziale Distanz geben, die zwischen Angestellten und Arbeitern einerseits, Kleinunternehmern andererseits bestand. Denn die familienfremden Arbeitskräfte erfuhren am eigenen Leib die Zwänge, unter die der Konkurrenzkampf die Meister oder Kaufleute setzte. Überlange Arbeitszeiten, schlechte Arbeitsbedingungen, knappe Bezahlung und genaue Überwachung machten - wie die Gewerkschaften kaum übertreibend formulierten - die Beschäftigten zu »Parias« der Lohnarbeiterschaft (Bonneff, 1911; Haupt, 1981, 112-141). Die in der zweiten Hälfte des 19. Jahrhunderts noch verbreitete Praxis, Kost und Logis zu stellen, schränkte die Handlungsfreiheit der Beschäftigten noch weiter ein. So waren in handwerklichen Berufen - wie in den Bäckereien - die miserablen Arbeitsbedingungen immer wieder Ursachen heftiger Arbeitskämpfe, die allerdings nur selten auf die Läden übergriffen (Haupt 1985). Die Streikhäufigkeit in handwerklichen Kleinbetrieben, aber auch die Zahl der Prozesse, die vor dem Conseils des Prudhommes stattfanden (David, 1974, 3-21, Zeldin, 1973, 1, 200-203), beweisen, daß die Beziehungen zwischen Meistern und Gesellen oder zwischen Ladeninhabern und Angestellten weder in der Mitte noch am Ende des 19. Jahrhunderts von jenem Vertrauen geprägt waren, das gesellschaftliche Harmonie suchende Autoren in ihnen ansiedelten. Obwohl die unterschiedlichen Interessen von Unternehmern und Lohnabhängigen Kleinbürger und Arbeiter voneinander trennten, beschränkte sich diese Trennung auf eine Minderheit der Klasse. Sie konnte in jenen Betrieben nicht entstehen, in denen keine Lohnarbeiter beschäftigt wurden oder in denen die Produktionsbedingungen der Meister und Prinzipale denen der Angestellten glichen. In einer Situation sozialer Gleichwertigkeit mit den familienfremden Arbeitskräften lebte die überwiegende Mehrheit der Händler und Handwerker (Gourden, 1983, $202 \mathrm{ff}$.).

Ein sozialer Erfahrungsraum, den zahlreiche Handwerker, Kleinhändler und Arbeiter mittlerer und großer Städte teilten, war das Stadtviertel, »le quartier«(Accampo, 1982, 39-52). In ihm knüpften sich enge Kontakte zwischen der Wohnbevölkerung und den Kleinbürgern, die teilweise über reine Geschäftsbeziehungen hinausgingen.

Vor 1914 blieben die Stadtviertel außerhalb des Zentrums noch die Bastionen kleiner Läden und Werkstätten, in denen - wie Alain Faure gezeigt hat - in geringen Mengen und auf Kredit an eine Kundschaft aus einfachen Kreisen verkauft wurde (Faure 1984). Die Struktur des Geschäftslebens ruhte dabei auf einem dichten Netz von Gesangsvereinen und Cafés, politischen Zirkeln und Geselligkeiten, in denen sich Verkäufer und Käufer wiedertrafen. Es ist kein Zufall, daß die »journées populaires«, in denen dieStadtbevölkerung im 19. Jahrhundert immer wieder ihren Unmut über zu hohe Preise und Steuern oder unzureichende Versorgung geäußert hatte, von den Stadtvierteln ausgingen (Rougerie, 1971). Politische Vergangen- 
heit, bestehende Formen der »sociabilité« und ökonomische Abhängigkeitsbeziehungen schmiedeten ein enges Band zwischen Kaufleuten, Handwerkern und Kunden.

$\mathrm{Zu}$ Recht hat Yves Lequin allerdings davor gewarnt, das Leben im Stadtviertel zu einem Idyll hochzustilisieren und hat auf die gewaltsamen Formen verwiesen, in denen auch zwischen Arbeitern und Kleinbürgern Konflikte ausgetragen wurden (Lequin, 1982, 3-7; Jaquemet, 1982, 66). Da die Kunden ebenso auf den Kredit, wie die Verkäufer auf ihre Kundschaft angewiesen waren, führte die Notwendigkeit zwar beide Teile immer wieder zusammen, schloß aber heftige Auseinandersetzungen über Preis und Qualität der Waren nicht aus. Schließlich waren auch die Positionen der Kaufleute und Handwerker nicht einheitlich. In Fragen, ob die Anwesenheit fliegender Händler zu erlauben sei, ob und wann die Läden zu schließen seien, brachen Divergenzen unter ihnen auf (Gourden, 1983, 324 ff.). Deshalb verfehlt ein monolithisches Bild des Stadtviertels die Realität. Half die Erinnerung in einem begrenzten geographischen Raum zweifellos dies Divergenzen im Kleinbürgertum abzuschwächen, so trug sie andererseits zur lokalistischen Fragmentierung der Klasse bei. Kirchliche Orientierung und Verankerung ging mit der Abwehr nationaler und weniger handgreiflicher Einflüsse einher und stärkte den »Lokalpatriotismus" (Hoffmann u.a., 1963, 1-117).

Vor allem im Heiratsverhalten und in den Beziehungen zu Arbeitskräften bestanden jedoch wichtige Unterschiede innerhalb des Kleinbürgertums. Im Unterschied zur Bourgeoisie, die sich seit Mitte des 19. Jahrhunderts zunehmend abgeschottet hatte, blieben Handwerk und Kleinhändler offen für Kontakte mit den Schichten der Arbeiterklasse. Wenn auch zunehmend härtere Auseinandersetzungen zwischen Kleinunternehmern und ihren Angestellten die Klassenlinie stärker betonten, so bildeten die proletarischen Bedingungen, unter denen zahlreiche Kleinhändler und Handwerker weiterhin lebten, doch eine Brücke zum Proletariat. Die Herausbildung des Kleinbürgertums als Klasse war vor 1914 offensichtlich unvollkommen.

In dem Begriff »classes moyennes« klang auch das Bild einer gesellschaftlichen Mittellage an. Danach lebten sie zwischen der Opulenz des Bürgertums und der Armut des Proletariats, zwischen einer stabilen und einer prekären Lebenssituation, zwischen Besitzanhäufung und -losigkeit und bildeten überdies eine Stufe in der gesellschaftlichen Mobilität. Untersucht man die Bedeutung des Kleinbürgertums für die soziale Mobilität, ihren Anteil an der Besitzverteilung und ihre Existenzsicherheit, so ist das Selbstverständnis der »Mittelklassen« zu nuancieren.

Untersuchungen der sozialen Mobilität bleiben solange problematisch, wie nicht geklärt ist, welche soziale Bedeutung jeder Beruf für Aufstieg und Deklassierung jeweils hatte. Diese allgemeine Problematik von Mobilitätsstudien erhält bei der Untersuchung des Kleinbürgertums ein besonderes Gewicht dadurch, daß sich hinter der Bezeichnung »Angestellte« sowohl eine Aufgabe der Unabhängigkeit als auch eine Wartestellung des Sohnes des Geschäftsinhabers verbergen kann. Dabei kann - je nach Situation des Geschäfts - die dauerhafte Angestelltenposition durchaus sozial höherwertig sein als die Übernahme des Ladens, dessen Kurzlebigkeit bekannt ist (Corbin, 1981, 109).

Trotz dieser Einschränkungen erlauben einige Mobilitätsuntersuchungen es doch, zumindest vorläufige Ergebnisse zu skizzieren. Insgesamt war die Öffnung nach »unten« größer als die nach »oben«. Handel und Handwerk bildeten im Paris der Jahrhundertmitte eine bevorzugte Anlaufstelle für Bauern und ländliche Handwerker, die das Land verließen. Profitierten in einigen Städten vornehmlich die Textilhandwerker von dieser Zuwanderung, so ließen sich in Paris vor allem die Bewohner der Auvergne im Handel und die aus dem Limousin im 
Bauhandwerk nieder (Raison-Jourdé, 1976). Nicht nur Migranten, sondern auch Mitglieder der Arbeiterklasse rückten in handwerk liche und kommerzielle Berufe ein. Vor der Februarrevolution war in Paris ein Fünftel der Ladenbesitzer zuvor als handwerklich qualifizierte Arbeiter tätig gewesen, und in der Parlamentsenquête des Jahres 1913 gaben die Korrespondenten auf die Frage nach der Berufserfahrung des Kleinhändlers 95 Arbeiter-, 92 Angestellten- und 53 Dienstbotentätigkeiten an (Enquête Landry; AN C 7463: Handelskammer Celle, 1913). Auch die Heimindustrie und die stravail à façon« waren für Arbeiter zugänglich, die sich bei einer günstigen Konjunkturlage selbständig machten, bei Krisen aber als Lohnarbeiter verdingten.

Dabei fand allerdings folgende Differenzierung in Handel und Handwerk statt: Während die wenig Kapital erfordernden Ladenöffnungen und handwerklichen Tätigkeiten weiterhin für "newcomer« offen blieben und eine Zone ständigen Austauschs zwischen Arbeitern und Kleinbürgern in den großen Städten bildeten, grenzte sich eine Gruppe besser situierter und stabiler Geschäfte und Werkstätten ab. Le Yaouang spricht deshalb für Paris in der Jahrhundertmitte von einer »andauernden Tendenz ... zur Selbstrekrutierung und des Rückzugs auf sich selbst«(Le Yaouang, 1979, 100). Eine ähnliche Tendenz zeichnete sich sowohl in Rouen ab, wo die Bewegung der gesellschaftlichen Verhältnisse ab 1850 fast zum Stillstand kam, als auch in Orléans um 1900, wo keine Aufstiegsmobilität stattfand(Chaline, 1970; Prost, 1981). Auf dem Land hingegen bewahrten Handel und Handwerk indes ihre Funktion als aufsteigende und als für den Aufstieg offene Klasse (Farcy, 1984).

Nur selten gelang von kleinbürgerlichen Berufen aus in der zweiten Hälfte des 19. Jahrhunderts der Sprung in das Bürgertum. Freilich stammten aus Handwerker- und Kleinhändlerkreisen Mitglieder des Bürgertums in Nancy wie auch hohe Beamte oder Lehrer. Bekanntlich war Léon Blum ja der Sohn eines Textilhändlers aus dem Zentrum von Paris. Der Königsweg des gesellschaftlichen Aufstiegs über die schulische Ausbildung, den die Radikalsozialisten dem Kleinbürgertum wies, stellte sich in der Wirklichkeit aber als eine enge Gasse heraus, durch die nur wenige Mitglieder der Klasse ihren Weg "nach oben« nehmen konnten (Voillard, 1982; Charle, 1980, 380-409; Prost, 1968, 362-364).

Handwerker und Kleinhändler gehörten formell zu der großen Besitzerklasse, die im Frankreich des 19. Jahrhunderts auf dem Land und in der Stadt das Rückgrat der bestenden Ordnung bildete. In der Besitzstruktur verloren sie aber im Laufe des 19. Jahrhunderts vor allem in den großen Städten an Gewicht, während sie auf dem Land offensichtlich ihre Positionen halten konnten. Bewahrten innerhalb von Handwerk und Kleinhandel auch einzelne Branchen stärker Anschluß an die allgemeine Besitzkonzentration, so gehörten beide bei der Besitzverteilung im 19. Jahrhundert jedoch zu den Verlierern: Gemessen an ihrer numerischen Bedeutung verfügten sie über zu wenig Kapital.

Gemäß dem Selbstverständnis der Mittelklassen hätten sie über eine zwar nicht ungefährdete, aber doch stabile Existenz verfügen müssen. Gegenüber dieser These fällt die Unsicherheit auf, die weite Teile des Handels und des Handwerks erfaßte. Diese zeigte sich besonders deutlich in den wirtschaftlichen Krisen, in denen die Kredite teurer wurden, die Kundschaft zahlungsschwächer und die Umsätze niedriger. Hatte die 1830er Krise in Paris noch stärker die größeren und mittleren Betriebe als die kleinen betroffen, so kehrte ab 1848 dieses Verhältnis um (Daumard, 1963, 428-439). Im Laufe der zweiten Hälfte des 19. Jahrhunderts schadeten Krisen stärker den kleinen Unternehmen und - verallgemeinert man die für Lyon in den 1880er Jahren ermittelten Ergebnisse - ruinierten eher die Läden als die Werkstätten (Bouvier, 1960, 256-257). 
Aber auch außerhalb der Krisenjahre war die Welt des Handwerks und des Handels in Bewegung. Freilich blieben Pleiten relativ selten, da sie auch den Verlust der Bürgerrechte nach sich zogen. Immerhin entfielen in den Jahren 1903-1905 26\% aller Pleiten auf Lebensmittelfabrikation und -handel und $16 \%$ auf die Herstellung und den Verkauf von Getränken. Allerdings meldeten damit nur 0,7\% aller Betriebe in diesen Branchen Konkurs an (Rapport Landry, 18). Umfangreicher waren die Geschäftsaufgaben, die vor einem formellen Konkursverfahren stattfanden. Vor allem Kleinst- und Kolonialwarenläden mußten verkaufen: $Z$ wei Drittel aller Verkäufe fielen vor 1914 auf diese niedrige Preisklasse: Je kleiner und kapitalschwächer mithin die Läden, desto höher war ihre Instabilität.

Von dieser Tendenz wichen in Paris allerdings die Obst- und Gemüsehändler ab. Die Fluktuation unter ihnen ging zwischen 1902 und 1912 nämlich von 17,5 auf 10,1\% zurück. Diese relative Stabilität in einer Branche, die durch einen niedrigen Kapitaleinsatz gekennzeichnet war, ist aus ihrer spezifischen sozialen Zusammensetzung zu erklären. Denn die Berechtigung einen fliegenden Handel mit Gemüse und Früchten zu eröffnen, vergab die Polizeipräfektur entweder an Männer über 50 Jahre oder an Frauen über 42 Jahre, die ansonsten von der Armenfürsorge hätten leben müssen. Da es für sie die letzte Möglichkeit war, eine halbwegs gesicherte Existenz zu führen, klammerten sich die meisten Gemüsehändler offensichtlich verbissener an ihr Geschäft als andere Detaillisten (Gourden, 1983, 282-289).

Wenn auch die Läden und Werkstätten der Dörfer und kleinerer Städte, sowie die Fachgeschäfte der großen Städte als Horte der Stabilität gelten können, so standen sie doch in einer Landschaft, in der Unsicherheit der Existenz eher die Regel als die Ausnahme war. Dieses Charakteristikum näherte Handwerker und Kleinhändler stärker den Arbeitern an, denen sie durch die Besitzlosigkeit, bzw. einen zur Formalie reduzierten Besitz der Produktionsmittel und durch die bestehenden Austauschbeziehungen ohnehin verbunden waren. Die soziale Welt des Handwerks und des Kleinhandels war gespalten zwischen jenen stabilen und traditionsreichen Ladenbesitzern und Meistern, die es vor allem auf dem Land und in kleinen Städten, aber auch in Großstädten zu materiellem Reichtum bringen konnten, den handwerklichen und kommerziellen Selbständigen, die einen qualifizierten Arbeiterberuf temporär oder dauerhaft für eine oft bedrohteSelbständigkeit aufgaben und den aus subproletarischem Milieu stammenden fliegenden Händlern und Heimarbeitern, für die Handel und Handwerk nicht mit dem Traum der Unabhängigkeit verbunden, sondern nur eine besondere Art war, ihr Überleben zu sichern. Aufstiegserwartungen und -möglichkeiten oder soziale Binnenorientierung trugen nur wenig zum Zusammenschluß dieser unterschiedlichen Teile bei. Auch das Zusammenleben im Stadtteil näherte eher die beiden letzten Gruppen einander an, als daß es zu einer »union sacrée« aller Ladenbesitzer und Handwerker geführt hätte. In der Gesellschaftsstruktur Frankreichs hielt das Kleinbürgertum keineswegs in allen seiner. Fraktionen eine Position der Mitte besetzt, sondern war mehrheitlich nach »unten« ausgerichtet. Es blieb deshalb in der französischen Gesellschaft des 19. Jahrhunderts, in der die soziale Durchlässigkeit zunehmend abnahm, »nur ein schmaler Durchgang zwischen dem Volk und dem gutsituierten Bürgertum.«(Chaline, 1970, 261).

\section{Politische Organisation und Differenzierungsprozesse der Mittelklassen}

Angesichts dieser ökonomischen und sozialen Vielfältigkeit erhält die Frage, ob politische Geschlossenheit des Kleinbürgertums die Spaltungen verhüllte, besonderes Gewicht. In der Tat deutet die breite Mittelklassenbewegung, die sich vor allem seit 1900 herausbildete, auf ei- 
ne hohe Kohäsion der Klasse hin. Im Unterschied zum 19. Jahrhundert, in dem Kleinhändler und Handwerker je nach Beruf und Ortschaft getrennt ihre Interessen verteidigten, nahmen mit dem Beginn des neuen Jahrhunderts die Verbände zu, die auf eine nationale und klassenübergreifende Organisation der Kleinbürger abhoben. Zu ihnen gehörte nicht nur die 1913 in 190 französischen Städten vertretene »Konföderation der französischen Handels- und Industriegruppen« sondern auch die kurzlebige »Französische Handels- und Industriepartei«, sowie seit 1907 auch die »Vereinigung zur Verteidigung der Mittelklassen«(Nord, 1981). Sie alle kennzeichnete der überregionale Zusammenschluß und das Bemühen, sowohl Handwerker als auch Kleinhändler anzusprechen. Dabei lag das Schwergewicht eindeutig im Handel, während die Angestellten ausgeschlossen blieben, die erst in der Krise der Volksfront des Jahres 1936 als Bündnispartner von konservativen Kräften umworben wurden (Boltanski, 1979, 75 bis 139; Kolboom, 1982, 71-96). Mit dem Zusammenschluß auf nationaler Ebene zogen die Mittelklassen die Konsequenz aus ihren gescheiterten Anläufen, ihre Forderungen in berufsspezifischen Organisationen oder in Interessengruppen zu vertreten, die in oder am Rande der republikanischen Partei operierten. Die Krisen in den 1880er und $1890 \mathrm{er} \mathrm{Jahren} \mathrm{hatten}$ die traditionelle Verbindung des Kleinbürgertums mit dem Republikanismus erschüttert und sie auf die Suche nach einer anderen politischen Heimat getrieben. Philip Nord hat überzeugend herausgearbeitet, daß sie über den Boulangismus, den sozialen Katholizismus bis hin zum Nationalismus und Poincarismus wanderten. Dabei rückten die in den 1880 er Jahren zwar schon existierende, aber sekundäre Xenophobie und die Abgrenzung vom Sozialismus in den Vordergrund einer Bewegung, die sich als politischer Faktor verstand und in der Abwehr staat licher Sozialgesetze und der Verteidigung des Privateigentums ihren ideologischen Zusammenhang fand. Ab 1910 schloß sie sich mit der Großindustrie zu einem mächtigen Interessenkartell zusammen (Nord, 1981). Während der Begriff »classes moyennes« in der Mitte des 19. Jahrhunderts an die bürgerliche Emanzipation erinnert hatte, füllte er sich vor dem 1. Weltkrieg mit konservativem Inhalt. Wie aus der Rede des Präsidenten der »Fédération nationale des Bâtiments et des Travaux publics« Villemin aus dem Jahre 1910 abzulesen ist, wurde er als Synonym für die Einheit des konservativen Lagers gegenüber der Revolution gebraucht. Leicht konnte das Lob der manuellen gegenüber der intellektuellen Arbeit betont, die wohltuende Wirkung des familiären Lebens gepriesen und folgendes Harmonienprinzip mit dem Begriff verbunden werden: »Für jede Sache braucht es einen Platz und jede Sache hat ihren Platz" (Etudes fiscales et sociales, 1911, 297). Geradezu mittelständischen Charakter nahm die Bewegung dadurch an, daß Kaufleute auch korporative Akzente in ihren Antworten auf die Enquête Landry setzten.

Schon die Forderung, den Siegeszug der Warenhäuser durch eine Begrenzung der von ihnen verkauften Warengruppen, der »spécialités«, zu stoppen, entstammte der zünftlerischen Tradition, den Absatz von Waren zu regulieren.

Häufiger waren die Attacken auf die Mängel staatlicher Politik, die es versäumt habe, eine zureichende Therapie für den Kleinhandel zu entwickeln und durch ungeschickte Gesetze dessen Situation in der Regel nur verschlechtere (AN C7640, Confédération des Groupes commerciaux et industriels de France, 1913). Die etatistische Ausrichtung der Bewegung war eindeutig stärker als ihre korporative.

Die Organisationen intervenierten massiv in das politische Leben Frankreichs und wurden sowohl von republikanischen als auch von konservativen Abgeordneten umworben. Ja, in Paris unterschieden sich nach 1910 die Wahlversprechen von Republikanern und Nationalisten nur wenig, da sie beide versuchten, die offensichtlich wahlentscheidenden Stimmen der 
Kleinhändler zu gewinnen (Raison-Jourde, 1976, 243-254). Eine genaue Analyse der Sozialgesetze, die in Frankreich vor allem zu Beginn des 20. Jahrhunderts verabschiedet wurden, erweist, daß die verschiedenen Regierungen ent weder bei der Formulierung der Gesetzestexte oder bei dem Erlaß der Durchführungsvorschriften die Interessen von Handwerk und Handel berücksichtigten und sie im Konfliktfall oft über die der Arbeiter stellten (Haupt, 1983). Dies alles weist darauf hin, daß es den Mittelklassen vor 1914 gelungen war, trotz deutlicher lokalistischer Tendenzen, im nationalisierten politischen Leben ihre Interessen durch nationale Organisationen geltend $\mathrm{zu}$ machen.

Erfaßten diese aber alle Kleinbürger und machten alle die Wende hin zum Poincarismus mit? Bereits die festgestellte hohe Fluktuation und Instabilität in Handel und Handwerk deuten darauf hin, daß die Organisierung dieser Klasse schwierig war, weil Seßhaftigkeit eine wichtige Voraussetzung für Widerstand und politischen Einfluß bildete. Nach Alain Corbin gehörten im Limousin Handwerker aufgrund ihrer langen Präsenz am Ort zu den politischen Meinungsmachern (Corbin, 1975, 301-307). Handwerker und Kleinhändler gehörten häufig zu den mobilen Teilen der Gesellschaft. In den von Kleinhändlern selbst genannten Zahlen jedoch nahm sich die Organisierung imposant aus: So gehörten in den 1880 er Jahren etwa $10 \%$ aller steuerpflichtigen Kleinhändler der »Ligue syndicale« an, und im Jahre 1912 waren 28 802, d.h. etwa ein Zehntel aller Krämer in 96 »chambres syndicales« zusammengeschlossen. Diese Angaben sind eher als Ausdruck einer politischen Praxis zu verstehen, den Umfang der Mittelklassenbewegung aufzublähen, denn als realistische Schätzung. Denn nach diesen Zahlen würde der Organisationsgrad im Kleinhandel über dem der Arbeiter in den Gewerkschaften liegen. Dies ist jedoch unwahrscheinlich (Nord, 1981, Annuaire des Syndicats professionnels, 1912). Mit dem Proletariat teilten die Kleinbürger jedoch die Kurzlebigkeit zahlreicher Verbände: 1912 waren von 96 Verbänden nur 27 älter als zehn Jahre. Im Unterschied zur Arbeiterklasse, in der die bei Streiks und Demonstrationen Mobilisierten zahlreicher waren als die Organisierten, ist bei den öffentlichen Aktionen abgeneigten Kleinbürgern anzunehmen, daß sie sich nicht außerhalb ihrer Organisationen manifestierten. Auch deshalb können die Ziele der Kleinbürgerorganisationen mit denen der Kleinbürger nicht gleichgesetzt werden.

Zahlreiche zeitgenössische Stellungnahmen verbreiteten nicht nur Klagen über die Schwierigkeiten, Kleinhändler und Handwerker zu organisieren, sondern unterstrichen auch, daß sich vor allem die begüterten Teile am Verbandsleben beteiligten. $\mathrm{Daß}$ vor allem relativ gesicherte Kleinhändler sich organisierten, läßt sich aus folgender Funktion ihres Zusammenschlusses ablesen. Angesichts der wiederholt vorgebrachten Klagen über die fehlende Qualifikation der kleinen Kaufleute ist er auch als Versuch zu interpretieren, die Professionalisierung des Gewerbes voranzutreiben und durch die Festlegung spezifischer Eignungsmerkmale die befähigten von den unbefähigten Kaufleuten zu trennen. An dieser Unterscheidung hatten vor allem die besser situierten Kaufleute Interesse (Gourden, 1983, 335-338). Auch bei den Stellungnahmen der Verbände standen die Interessen der Lohnarbeiter beschäftigten Kleinbürger eher im Vordergrund als die der Familienbetriebe. Versuchten sie auch durch allgemeine Parolen eine möglichst breite Interessenallianz zu erstellen, so optierten sie im Konfliktfall für die Ziele der besitzenden Teile der Klasse.

Weiterhin ist der Umfang der Wendung zum Konservatismus im Kleinhandel nicht zu überschätzen. Nach den Hinweisen von Jeanne Gaillard lebte der kleinbürgerliche Republikanismus stärker in den Provinzen als in Paris fort. Auch vollzog sich zwischen Kleinbürgertum und Sozialismus kein radikaler Bruch nach 1900. Nicht nur bestanden bei Streiks Interessenkoalitionen zwischen sozialistischen Arbeitern, Kleinhändlern und Handwerkern fort (Per- 
rot, 1974, 281 f., 488 f., 535 f.), sondern in der sozialistischen Partei arbeiteten weiterhin Kleinbürger mit. So gehörten ein Schneidermeister, ein Kleinindustrieller und ein Weinhändler zu den sozialistischen Führern im westfranzösischen Departement Isère. Aus der starken Vertretung von Handwerkern und Kleinhändlern und den Kadern der SFIO im Loir-et-Cher schlußfolgert G. Dupeux sogar: »Der Sozialismus wurde im Departement Loiret-Cher vom Kleinbürgertum verankert.« (Dupeux, 1972, 608; Barral, 1962, 195)

Schließlich bot die Rhetorik der Kleinhändlerorganisationen ebenso wie die der Sozialisten Anknü pfungspunkte für eine Zusammenarbeit. In den Chor der antisemitischen, nationalistischen und korporativen Stimmen mischten sich 1913 auch jakobinische Klänge, die an das Engagement von Handwerkern und Kleinhändlern in den Revolutionen des 19. Jahrhunderts erinnerten. Antiadelige, egalitäre und liberale Argumente gingen dabei eine Verbindung ein. Eine Organisation verband die Durchsetzung der Warenhäuser mit dem Sieg einer neuen Aristokratie: »Dort sah man eine neue und überaus mächtige reich: die kapitalistische Aristokratie, die der ganzen Nation diente.« (AN C 7468, Fédération du commerce et de l'industrie de l'arrondissement de Castres, 1913). In einer Aufnahme der Begrifflichkeit der Sanskulotten wurden die großbetrieblichen Organisationen zu $» \mathrm{Mo}$ nopolen« und »Wucherern«, die es zu bekämpfen gelte (AN C 7461 Chambre de Commerce de Bourges(Cher)). Ganz im Stil des Jahres 1793 schrieb das Syndicat des épiciers au détail aus Nancy die Verallgemeinerung des Kleinbürgertums auf ihre Fahnen:

"Es ist, im Gegenteil, von allgemeinem Interesse, daß die Staatsmacht den Zugang zum Eigentum für die größtmögliche Anzahl von Eigentümern fördert. Das ist der Beweis des Fortschritts, der Zivilisation und der Freiheit. Die Revolution von 1789 wurde mit dem Ruf: "Es lebe die Freiheit« gemacht! Sollen wir nun schlapp und degeneriert werden und uns diese Freiheit rauben lassen? (AN C 7465)

Wenn dieSozialisten auch die These vom Niedergang des Kleinbesitzes vertraten, eine Verallgemeinerung des Eigentums ablehnten und für dessen Organisation in Genossenschaften warben, so konnten sie ihre Verherrlichung der französischen Revolution doch mit dem Jakobinismus einiger Kleinhändlerorganisationen in Einklang bringen(Rébérioux, 1981, 57-70). Überdies bot ihnen die Interpretation der Lage der Kleinbürger, deren $\gg$ so unsichere als auch miserable Lage« Jaurès beklagte, die Möglichkeit, sie als Ausgebeutete gegen das Großkapital zu verteidigen (zit. in Gaumont, 1923, II, 285). In diesem Zusammenhang ist auch die Initiative der sozialistischen Parlamentsfraktion für eine Enquête über den Kleinhandel in Frankreich zu verstehen. Freilich stand der dogmatische Flügel der SFIO unter Jules Guesde einer Zusammenarbeit mit den Mittelklassen feindlich gegenüber, aber die Parteimitte bezog mehrfach vor 1914 die Zusammenarbeit mit der Partei der Kleinbürger, den Radikalsozialisten, in ihr politisches Kalkül ein. Obwohl Ende des 19. Jhds. die Konflikte zwischen Arbeitern und Kleinkapitalisten an Schärfe gewannen, konnten die Kleinbürger, die keine familienfremden Arbeitskräfte beschäftigten, sehr wohl mit der SFIO sympathisieren.

Die lautstarke und erfolgreiche Agitation der Kleinbürgerorganisationen kann nicht verdekken, daß der wirtschaftlichen und sozialen Vielfalt der Klasse auch eine politische Buntschekkigkeit entsprach. Ein generell geringer Organisations- und Mobilisierungsgrad bei relativ stärkeren Aktivitäten der besser situierten Handwerker und Kleinhändler, das Nebeneinander von ständisch reaktionärer, konservativer, republikanischer und gar sozialistischer Ausrichtung machten die Differenziertheit aus. Freilich ist damit die oft wiederholte Annahme nicht widerlegt, daß gerade, wenn sich die Lebensbedingungen von Klassen einander annäherten, diese sich politisch oder ideologisch von einander distanzierten. Ob sich in Frankreich etwa die Kleinbürger von den Arbeitern absetzten, denen sie oft sehr nahe standen, 
kann nur eine Analyse der kulturellen Ausdrucksformen, der Lebensweise und des Geschmacks klären, die Pierre Bourdieu für die Gegenwart vorgelegt hat, die aber für die Zeit vor 1914 noch fehlt (Bourdieu, 1979).

Der Versuch, Lage und das Verhalten von Kleinhandel und Handwerk in Frankreich vor 1914 mit dem Begriff »Mittelklassen« zu analysieren, hat den Vorteil, daß das Selbstverständnis der Handelnden einbezogen wird. Im Unterschied zu Ansätzen, die ein starres sozioökonomisches Gerüst als ausreichend ansehen, um Verhalten abzuleiten, können die Selbstaussagen von geschichtlichen Akteuren, kann die Ebene des Diskurses Widersprüchlichkeiten und Tendenzen auf ideologischer Ebene erfassen helfen. Allerdings gewinnnen diese Untersuchungen zweifellos dadurch, wenn sie durch die Erkenntnisse der Klassenanalyse korrigiert werden. Diese ergeben, daß die Verlautbarungen der Mittelklassenorganisationen in Frankreich vor 1914 keineswegs als repräsentativ für die Gesamtheit der Kleinbürger gelten. Trotz dieses Vorteils besitzt der Begriff "Mittelklassen« eine geringe analytische Kraft. Allein von seinen ideologischen Konnationen her siedelt er Handwerk und Kleinhandel in der Mitte der Gesellschaft, im »juste milieu« an, zu dessen Träger und Verteidiger sie werden. In ihn gehen deshalb ungebrochen und undiskutiert Vorstellungen von gesellschaftlicher Normalität ein. Generell betont er zu sehr politische und ideologische Zielsetzungen, um sich als tragfähiges Konzept für eine Untersuchung der Klassenlage und -beziehungen zu erweisen. Die Studien, die mit ihm operieren, setzen alle bezeichnenderweise auf den politischen Bereich und verabsolutieren damit einen Aspekt der Klassenwirklichkeit.

Ein weiterer Mangel der Mittelklassenthese liegt darin, daß sie Handwerk und Kleinhandel durch zwei andere Klassen: die Arbeiterklasse und die Bourgeoisie begrenzt. Wie notwendig für die Untersuchung auch der Rekurs auf eine der beiden »Hauptklassen« ist, so muß doch bewußt sein, daß diese nach dem Prinzip konstruiert sind, die Realität dem Begriff unterzuordnen und nicht etwa die Realität auf den Begriff zu bringen. Analog zu den Differenzierungen im Kleinbürgertum wäre auch nach Verwerfungen in der Arbeiterklasse und im Bürgertum zu fragen. Schon allein der Hinweis, daß sowohl von den Facharbeitern, aber auch von den subproletarischen Teilen und den im Stücklohn Beschäftigten, Übergangsmöglichkeiten zurSelbständigkeit bestanden, wirft ein Schlaglicht auf die Unterschiedlichkeit innerhalb des Proletariats.

Im Gegensatz zu dieser Klassendefinition geht eine andere oft von einem feststehenden Klassenbegriff aus, der in dem gemeinsamen Besitz der Produktionsmittel seine ökonomische Basis und in dem sich aus diesem entwickelnden Klassenbewußtsein seine ideologische Fortsetzung hat. Wie verfeinert dieses Modell inzwischen auch ist, so bietet es zwar die Möglichkeit, den Abstand zwischen der historischen Realität "Kleinbürgertum» und dem Begriff »Klasse" zu ermitteln. Aber es unterstellt, als gäbe es eine allgemeine, jederzeit gültige Klassenbestimmung, die unabhängig von Ort und Zeit in der Analyse historischer Strukturen und Prozesse "anzuwenden« sei. Gerade das Beispiel des Kleinbürgertums zeigt aber, wie notwendig es ist, keine allgemeine Klassentheorie der empirischen Untersuchung überzustülpen, sondern diese jeweils neu zu gewinnen. In diese muß eingebracht werden, daß die Bedeutung des Produktionsmittelbesitzes für die Klassenhaltung geringer war als die finanziellen Abhängigkeiten, daß die soziale Instabilität prägender als die Stellung in der Gesamtgesellschaft, die Geselligkeit einender war als formelle Organisationen und die lokale oft ebenso wichtig wie die nationale Erfahrung war. Durch eine derartige Vorgehensweise gewinnt nicht nur die Theoriebildung, sondern auch die Durchdringung der historischen Wirklichkeit. 


\section{Literatur}

E. Accampo 1982, 'Entre la classe sociale et la cité: identité et intégration chez les ouvriers de SaintChamond, 1815-1880', in: Le Mouvement social 118, S. 39-59

AN C 7461, 7463, 7465, 7466, 7467, 7468, 7470, 7640, 13267 (Archives Nationales, Serie C)

Annuaire des syndicats professionels, Paris 1912

Annuaire statistique de la ville de Paris, 1900-1914. Résultat des ventes des fonds de commerce.

A. Armengaud 1961, Les Populations de l'est-acquitain au début de l'époque contemporaine. Recherches sur une région moins développée (vers 1845-vers 1871), Paris.

P. Barral 1962, La Département de l'Isère sous la Troisième République 1870-1940, Histoire sociale et politique, Paris.

P. Barral (Hg.) 1968, Les Fondateurs de la troisième république, Paris.

E. Bloch 1973, Erbschaft dieser Zeit, Frankfurt/Main.

L. Boltanski 1979, 'Taxinomies sociales et luttes de classes. La mobilisation de la classe moyenne et l'invention des "cadres«, in: Actes de la recherche en sciences sociales., 5. 75-105.

L. und M. Bonneff 1911, La vie tragique de travailleurs. Enquête sur la condition économique et morale des ouvriers et ouvrières d'industrie, Paris.

P. Bourdieu 1979, La Distinction. Critique sociale du jugement, Paris.

J. Bouvier 1960, Le Krach de l'union général 1878-1885, Paris.

J.P. Chaline 1970, 'Les contrats de mariage à Rouen au XIXe siècle. Etude d'après l'enregistrement des actes civils publics', in: Revue d'histoire économique et sociale 48, S. 260-4.

C. Charle 1980, 'Le recrutement des grands fonctionnaires en 1901', in: Annales ESC, S. 380-409.

A. Corbin 1981, La mobilité sociale en France au XIVe siècle, in: G.A. Ritter und R. Nierhaus (Hg.), Aspekte der historischen Forschung in Frankreich und Deutschland, Göttingen.

A. Cottereau 1980, 'Introduction' zu: D. Poulot, Le Sublime ou le travailleur comme il est en 1870 et ce qu'il peut être, Paris.

A. Daumard 1963, La Bourgoisie parisienne de 1815 à 1848, Paris.

M. David 1974, 'L'Evolution historique des Conseils de prud'hommes en France', in: Droit social, Februar, S. 3-21.

J. Dubois 1962, Le Vocabulaire politique et social en France de 1868 à 1872, Paris.

J.C. Farcy 1984, Rural artisans in the Beauce during the nineteenth century, in: G. Crossick/ H.G. Haupt (Hg.), Shopkeeper and artisans in nineteenth Century Europa, London-New York, 219-238.

A. Faure 1980, 'Note sur la petite entreprise en France au XIXe siècle. Réprésentation d'état et réalité, Papier vorgetragen auf dem jährlichen Kongreß der französischen Wirtschaftshistoriker, Paris.

A. Faure 1984, The grocery trade in nineteenth century Paris: a fragmented corporation, in: Crossick/ Haupt (Hg.) 155-174.

M. Fischer 1974, Mittelklasse als politischer Begriff in Frankreich seit der Revolution, Göttingen.

C. Fohlen 1956, L'Industrie textile au Sécond Empire, Paris.

J. Gaillard 1977, Paris, la ville 1852-1870, Paris.

J. Gaumont 1923, Histoire générale de la coopération en France, Paris.

P. Gemahling 1912, 'La concentration commerciale sans grands magasins', in: Revue d'économie politique, 26 , S. 170-192.

J.M. Gourden 1983, 'Les petits métiers parisiens et leurs fonctions au XIXe siècle. L'example des marchands des quatre saisons', Thèse 3e cycle, Université Paris VII.

P. Guillaume 1972, La Population de Bordeaux aux XIXe siécle, Paris.

H.-G. Haupt 1981, 'Angestellte in der französischen Gesellschaft vor 1914. Einige einführende Bemerkungen', in: J. Kocka (Hg.), Angestellte im europäischen Vergleich. Die Herausbildung angestellter Mittelschichten seit dem späten 19. Jahrhundert, Göttingen, S. 112-141.

H.-G. Haupt 1983, La politique sociale.

H.-G. Haupt 1985, 'Les petits commerçants et la politique sociale. Etude sur l'application du repos heb- 
domadaire en France et en Allemagne avant 1914', in: La petite entreprise en France, Paris.

S. Hoffmann et.al. 1963, In Search of France, Cambridge.

G. Jacquement 1982, 'Belleville ouvrier à la Belle époque', in: Le Mouvement social 118, S. 62-77.

C.H. Johnson 1979, 'Patterns of proletarianization: Parisien tailors and Lodève woollen workers', in: J. Merriman ( $\mathrm{Hg}$.), Consciousness and Class Experience in Nineteenth Century Europe, New York, S. 65-84.

J. Kocka (Hg.) 1983, Europäische Arbeiterbewegungen im 19. Jahrhundert, Göttingen.

J. Kolboom 1982, 'Patronat et Cadres: la contribution patronale à la formation du groupe des cadres (1936-1938)', in: Le Mouvement social 121, S. 71-96.

A. Landry 1914, Rapport fait au nom de la commission du commerce et de l'industrie chargée de procéder à une enquête sur la situation du commerce en France et notamment sur la condition actuelle du petit commerce, Chambre des Députés, 10ème Législature, 85 impression 3432.

A. Leppert-Fogen 1973, Die deklassierte Klasse. Studien zur Geschichte und Ideologie des Kleinbürgertums, Frankfurt/Main. .

Y. Lequin 1982, 'Ouvriers dans la vile (XIXe et XXe siècle), in: Le Mouvement social 118, S. 3-7.

J. Le Yaouang 1979, 'La mobilité sociale dans le milieu boutiquier parisien au XIXe siècle, in: Le Mouvement social 108, S. 89-112.

J. Le Yaouang 1981, 'Aux origines du milieu boutiquier parisien au XIXe siècle. La nuptialité et ses aspects sociaux', unveröffentlichtes Papier, vorgetragen bei der 3. Gesprächsrunde über das europäische Kleinbürgertum im neunzehnten Jahrhundert an der Universität von Paris X., Nanterre.

P. Nord 1981, 'Commerce and Culture in fin de siècle Paris', PhD thesis, Columbia University.

P. O’Brien/C. Keyder 1978, Economic growth in Britain and France 1780-1914: Two Paths to the Twentieth Century, London.

M. Perrot 1974, Les Ouvriers en grève, France 1871-1890, Paris.

A. Prost 1968, L'enseignement en France 1800-1967, Paris.

A. Prost 1981, 'Mariage, jeunesse et société à Orléans en 1911', in: Annales ESC 36, S. 679-83.

F. Raison-Jourdé 1976, La Colonie auvergnate de Paris au XIXe siècle, Paris.

M. Rebérioux 1981, 'Les socialistes français et le petit commerce au tournant du siècle, in: Le Mouvement social 114, S. 57-70.

J. Rougerie 1971, Paris libre 1871, Paris.

M. Saint-Léon 1911, in: Musée Social, S. 372.

J. Scott 1984, 'Men and Women in the Parisian garment trades; discussions of family and work in the 1830s and 1840s, in: P. Thane, G. Crossick and R. Floud (eds.), The Power of the Past. Essays for Eric Hobsbourn, Cambridge.

A. de Tocqueville, L'Ancien Régime et la révolution, in: Oeuvres complètes, II, 1.

Villemin 1911, Discours, in: Etudes fiscales et sociales.

O. Voilliard 1982, Nancy au XIXe siècle (1815-1871), une bourgeoisie urbaine, Paris.

H.A. Winkler 1976, 'From Social Protectionism to National Socialism. The German Small-Business Movement in Comparative Perspective, in: Journal of Modern History 48, S. 1-18.

T. Zeldin 1973, France, 1848-1945, Oxford. 